\title{
CONTROLLABLE PREPARATION OF NANO MOLYBDENUM DISULFIDE BY HYDROTHERMAL METHOD
}

\author{
"SUO XIA HOU, CHAO WU*, YING JIE HUO \\ College of Mechanical Chemical Engineering, North China University of Science and Technology, \\ Tangshan Hebei, 063009, China \\ *College of Mechanical Engineering, Hunan University of Arts and Science, \\ Changde, Hunan, 415006, China \\ \#E-mail: kyccgk@ncst.edu.cn, housuoxia@163.com
}

Submitted September 22, 2016; accepted January 10, 2017

Keywords: Nano molybdenum disulfide, Hydrothermal method, Nanoflowers, Nanospheres

\begin{abstract}
Nano molybdenum disulfide possesses unique chemical and physical properties. In this paper molybdenum disulfide nanoparticles with spherical and flower-like structure are prepared via a hydrothermal method. Sodium molybdate and thioacetamide are taken as precursors, polyethylene glycol (PEG-20000), hexadecyl trimethyl ammonium chloride (CTAC) and anhydrous ethanol are used as additives. The properties of the product are characterized by X-ray diffraction (XRD) and scanning electron microscopy (SEM). The results showed that under acidic conditions, molybdenum disulfide nanoparticles with spherical shape are obtained when PEG-20000 and CTAC are added. The nanoparticles are uniform in size with a diameter of about $100 \mathrm{~nm}$. Molybdenum disulfide nanoparticles with a flower-like structure are obtained when anhydrous ethanol is added. Their diameters under sulfuric acid and hydrochloric acid conditions are $190 \mathrm{~nm}$ and $70 \mathrm{~nm}$, respectively. Yield analysis reveals that the highest yield (which can be up to $79 \%$ ) occurs by adding polyethylene glycol in a sulfuric acid environment.
\end{abstract}

\section{INTRODUCTION}

Transition metal binary compounds with layered structure possess unique properties in lubrication [1], catalysis [2], photoelectric devices and other fields [3], and have become a hot research topic [4-7]. Molybdenum disulfide $\left(\mathrm{MoS}_{2}\right)$ is a black powder with metallic luster in the normal condition. It is a typical transition metal binary compound with diamagnetic and semiconducting properties, excellent antifriction performance and outstanding abrasion resistance because of its unique structural characteristics. It is widely used for lubricants in equipment manufacturing and industrial manufacture.

$\mathrm{MoS}_{2}$ nanoparticles are small in size, which facilitates penetration into friction pair surfaces and improves their adsorption properties. They are superior to conventional $\mathrm{MoS}_{2}$ in antifriction performance, abrasion resistance and extreme pressure performance [8]. Various forms of $\mathrm{MoS}_{2}$, including inorganic fullerene structures [9], nanowires [10], nanotubes [11-12], nanorods [13], nanospheres [14], hollow spheres [15] and nanoflowers [16], have been successfully prepared recently. However, problems of poor reproducibility of preparation and low product quality and yield still exist. Therefore, looking for a robust and inexpensive synthetic method is a primary aim of current research. Compared to other synthesis methods for $\mathrm{MoS}_{2}$, chemical synthesis can produce sulfides with high purity, fewer impurities and fine granularity [17].

In this paper, $\mathrm{MoS}_{2}$ is synthesized via a chemical synthesis method in which sodium molybdate and thioacetamide are taken as precursors, and polyethylene glycol (PEG-20000), anhydrous ethanol or hexadecyl trimethyl ammonium chloride (CTAC) are chosen as additives. The morphology of the synthesized molybdenum disulfide is analyzed and compared by using scanning electron microscopy (SEM) and the influence of the mechanism and additives on the morphology of $\mathrm{MoS}_{2}$ are discussed.

\section{Experimental}

\section{Reagents and equipment used}

The reagents used in this work were sodium molybdate, thioacetamide, PEG-20000, CTAC, absolute ethyl alcohol, hydrochloric acid (15 wt. \%) and sulfuric acid (98 wt. \%). The experimental equipment used is listed in Table 1. 
Table 1. Experimental equipment used in this work.

\begin{tabular}{lll}
\hline Name & Model & Manufacturer \\
\hline Muffle furnace & BSX2-6-12TP & Shanghai Yiheng \\
\hline Centrifuge machine & H2050 & Shanghai Luxiangyi \\
\hline $\begin{array}{l}\text { Electric thermostatic } \\
\text { drying Oven }\end{array}$ & GZX-9070MBE & Shanghai Boxun \\
\hline $\begin{array}{l}\text { Scanning electron } \\
\text { microscope }\end{array}$ & S-4800 & Hitachi \\
\hline X-ray spectrometer & D/MAX2500PC & Rigaku Corporation \\
\hline
\end{tabular}

\section{Sample preparation}

Amounts of $0.3 \mathrm{~g}$ sodium molybdate and $0.55 \mathrm{~g}$ thioacetamide were weighed in on an electronic balance and put into a polytetrafluoroethylene (PTFE) lining before adding $1.44 \mathrm{~g}$ of polyethylene glycol (or $0.05 \mathrm{~g}$ of CTAC or $5 \mathrm{ml}$ of absolute ethyl alcohol) and $50 \mathrm{ml}$ of deionized water. Next, the mixture was homogenized by stirring, and either hydrochloric acid or sulfuric acid was added dropwise in order to set the $\mathrm{pH}$ of the solutions to 1.0 while stirring. After sufficient reaction, the mixtures were given into an autoclave, which was placed into a muffle furnace for $6 \mathrm{~h}$, keeping the temperature at $220^{\circ} \mathrm{C}$. The mixtures were repeatedly washed with deionized water in a centrifuge until neutral $\mathrm{pH}$ was achieved, and then put into a drying box. After drying the solid residues were weighed. Finally, the precipitate was ground. The main reaction equations and the flow chart (Figure 1) are as follows.

$$
\begin{gathered}
\mathrm{CH}_{3} \mathrm{CSNH}_{2}+2 \mathrm{H}_{2} \mathrm{O} \rightarrow \mathrm{CH}_{3} \mathrm{CONH}_{4}+\mathrm{H}_{2} \mathrm{~S} \\
\mathrm{Na}_{2} \mathrm{MoO}_{4}+4 \mathrm{H}_{2} \mathrm{~S} \rightarrow \mathrm{Na}_{2} \mathrm{MoS}_{4}+4 \mathrm{H}_{2} \mathrm{O} \\
\mathrm{Na}_{2} \mathrm{MoS}_{4}+4 \mathrm{HCl} \rightarrow \mathrm{H}_{2} \mathrm{MoS}_{4}+2 \mathrm{NaCl} \\
\mathrm{Na}_{2} \mathrm{MoS}_{4}+\mathrm{H}_{2} \mathrm{SO}_{4} \rightarrow \mathrm{H}_{2} \mathrm{MoS}_{4}+\mathrm{Na}_{2} \mathrm{SO}_{4} \\
\mathrm{H}_{2} \mathrm{MoS}_{4} \rightarrow \mathrm{MoS}_{3} \downarrow+\mathrm{H}_{2} \mathrm{~S} \\
\mathrm{MoS}_{3} \stackrel{800{ }^{\circ} \mathrm{C}}{\longrightarrow} \mathrm{MoS}_{2} \downarrow+\mathrm{S}
\end{gathered}
$$

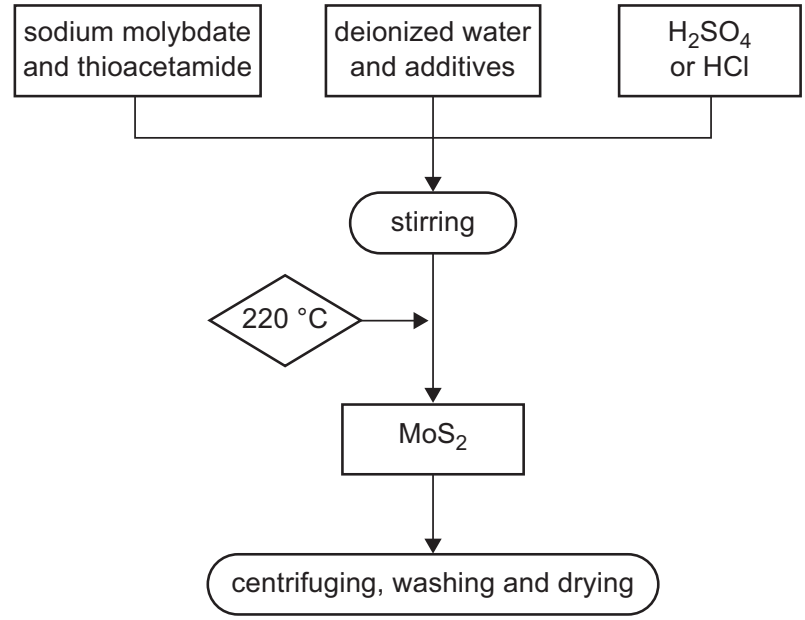

Figure 1. Process flow chart for preparing $\mathrm{MoS}_{2}$.

\section{RESULTS AND DISCUSSION}

\section{Characterization of molybdenum disulfide via XRD}

The crystal structure of the sample was characterized by X-ray diffraction (XRD). As shown in Figure 2, all XRD peaks can be indexed by cards of the standard powder diffraction of $\mathrm{MoS}_{2}$. The four main peaks match the standard diffraction peaks on card (JCPDS 37-1492), which indicates that the samples are hexagonal phase $\mathrm{MoS}_{2}$. The diffraction peak (002) of $\mathrm{MoS}_{2}$ prepared under the two kinds of acidic conditions is high and sharp in both cases, which illustrates that the layers are stacked well when $\mathrm{MoS}_{2}$ is synthesized via this hydrothermal method.

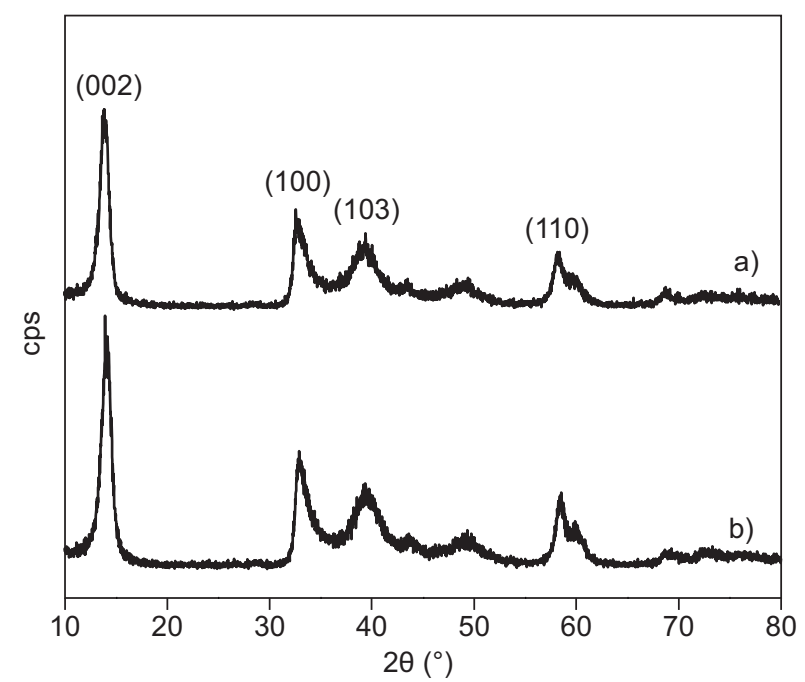

Figure 2. XRD patterns of $\mathrm{MoS}_{2}$ samples prepared in: a) sulfuric acid condition and $\mathrm{b}$ ) hydrochloric acid condition.

Study on the morphology of nano molybdenum disulfide

Figure 3 shows the SEM micrographs of $\mathrm{MoS}_{2}$ synthesized using different additives under sulfuric acid condition (Figure $3 \mathrm{a}-$ nano $\mathrm{MoS}_{2}$ with spherical structure and a diameter of $90 \mathrm{~nm}$ prepared by adding PEG-20000, Figure $3 \mathrm{~b}$ - nano $\mathrm{MoS}_{2}$ with spherical structure and a diameter of $110 \mathrm{~nm}$ prepared by adding CTAC, Figure $3 \mathrm{c}$ - nano $\mathrm{MoS}_{2}$ with flower-like structure and a diameter of $190 \mathrm{~nm}$ prepared by adding absolute ethyl alcohol). Figure 4 shows the corresponding SEM micrographs of $\mathrm{MoS}_{2}$ synthesized using different additives under hydrochloric acid condition (Figure 4a - nano $\mathrm{MoS}_{2}$ with spherical structure and a diameter of $105 \mathrm{~nm}$ prepared by adding PEG-20000, Figure 4b - nano $\mathrm{MoS}_{2}$ with spherical structure and a diameter of $120 \mathrm{~nm}$ prepared by adding CTAC, Figure $4 \mathrm{c}-$ nano $\mathrm{MoS}_{2}$ with flower-like structure and a diameter of $70 \mathrm{~nm}$ prepared by adding absolute ethyl alcohol). 


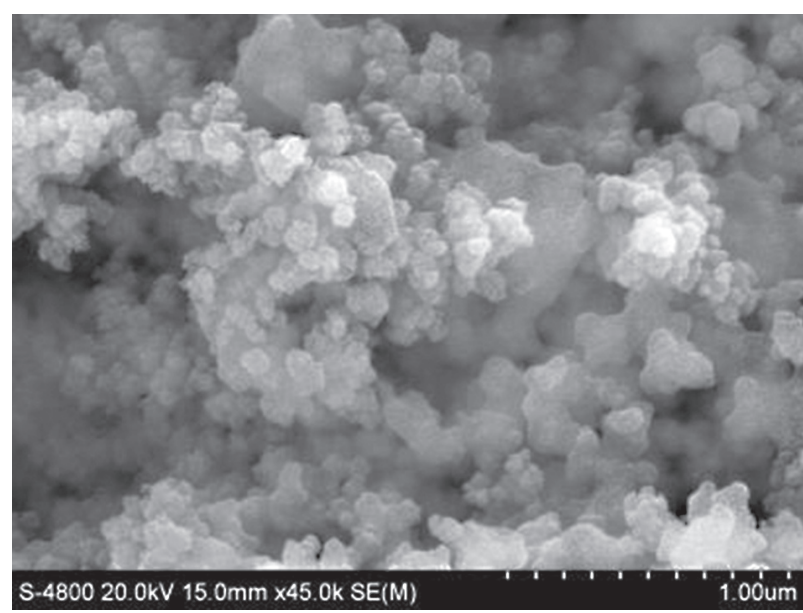

a) PEG-20000

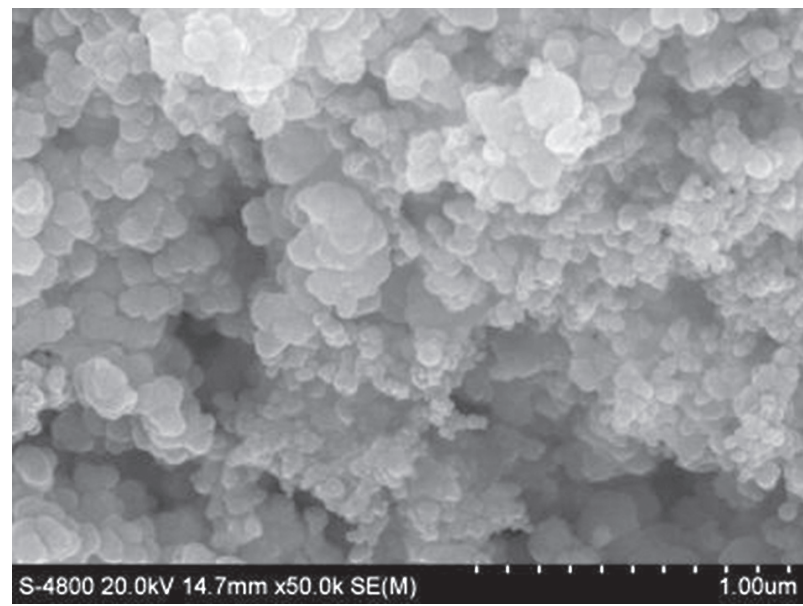

b) CTAC

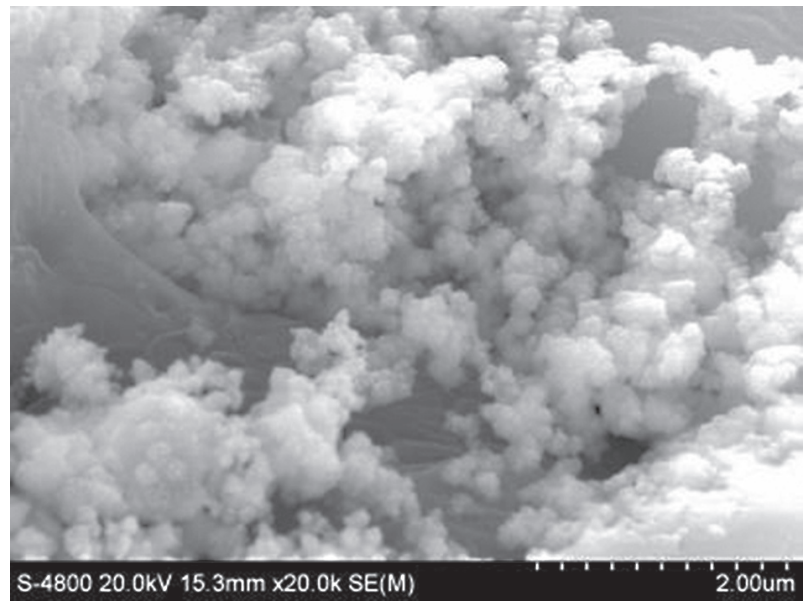

c) Anhydrous ethanol

Figure 3. SEM micrographs of $\mathrm{MoS}_{2}$ synthesized using different additives under sulfuric acid condition.

The influence mechanism of additives on $\mathrm{MoS}_{2}$

Surfactants, amphipathic molecules composed of hydrophilic and oleophobic polar groups and hydrophilic and oleophobic non-polar groups, play an important role

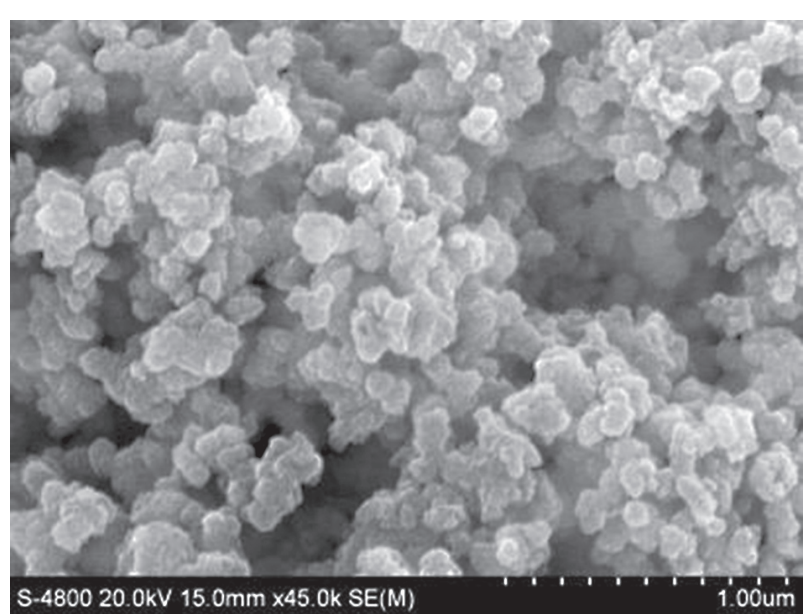

a) PEG-20000

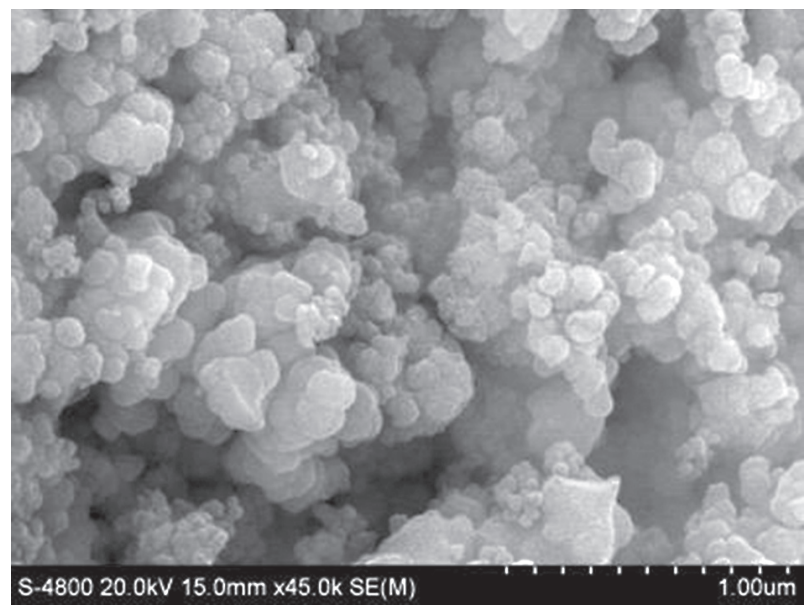

b) CTAC

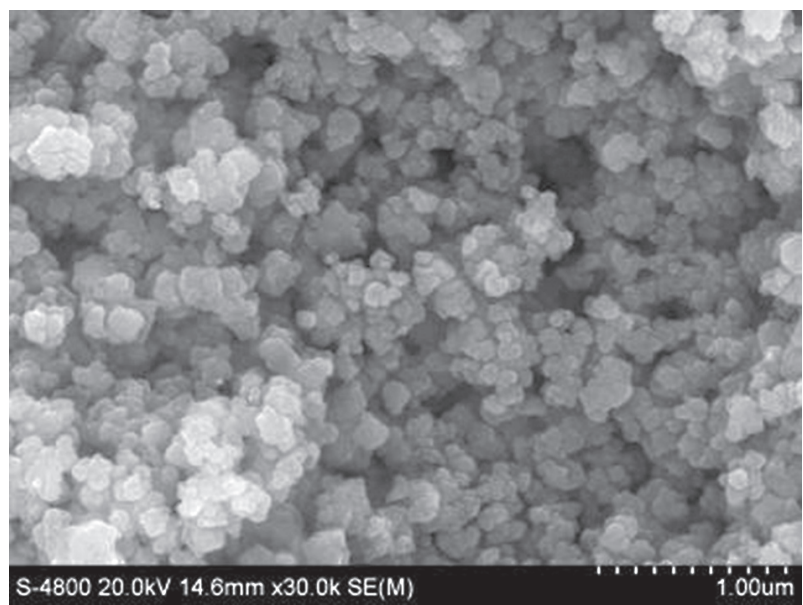

c) Anhydrous ethanol

Figure 4. SEM micrographs of $\mathrm{MoS}_{2}$ synthesized using different additives under hydrochloric acid condition.

in the synthesis of nanoparticles. They can be classified into ionic and non-ionic types. Moreover, depending on the type of ions, the former can be divided into cationic, anionic and amphiprotic surfactants. When the surfactants concentration exceeds a critical value, they start to aggregate and form micelles of different shapes [18]. 
Surfactants act on the surface of solid particles and affect the process of grain growth when $\mathrm{MoS}_{2}$ is being prepared, so they are often used for improving the dispersity of $\mathrm{MoS}_{2}$, relieving the aggregation of particles and as an auxiliary tool for grain morphology control [19]. Polyethylene glycol with the molecular formula $\mathrm{HO}\left(\mathrm{C}_{2} \mathrm{H}_{4} \mathrm{O}\right)_{n} \mathrm{H}$ belongs to non-ionic type surfactants. Its solubility is high in water, because the molecular chain adopts the shape of a snake in water, with two kinds of hydrophilic groups, ether and hydroxyl, and no hydrophobic groups. The long chain of this high-molecular-weight polymer is attracted to the surface of dispersed grains, which forms a layer of macromolecular hydrophilic membrane and is responsible for strong excluded volume effects ("stereo-hindrance"). Therefore the repulsive energy between the particles is further increased to prevent agglomeration. Besides, the dispersibility and stability of the system are enhanced, the growth of the previously formed nuclei is restrained, and nanocrystals of $\mathrm{MoS}_{2}$ are effectively dispersed. With the aid of the organic macromolecular chain of high concentration, the $\mathrm{MoS}_{2}$ nanocrystals are coated and modified [20]. Moreover, the modification with cationic CTAC has a good effect on the formation of $\mathrm{MoS}_{2}$ with spherical structure. It is mainly because of the negative electric charge on the surface of the $\mathrm{MoS}_{2}$ nanoparticles due to the electrostatic interaction that surfactants of cationic type with positive electricity are selectively attracted on the surface of particles (so-called specific adsorption). This not only reduces the surface tension of the particles, but also effectively prevents the agglomeration of particles by means of the "stereohindrance", thereby a highly dispersed nano $\mathrm{MoS}_{2}$ with spherical structure of low surface activity is synthetized [21]. The effects of anhydrous ethanol as a dispersant are similar to those of polyethylene glycol. Nano $\mathrm{MoS}_{2}$ with flower-like structure is synthetized when absolute ethyl alcohol is used. Stereo-hindrance is related to the length

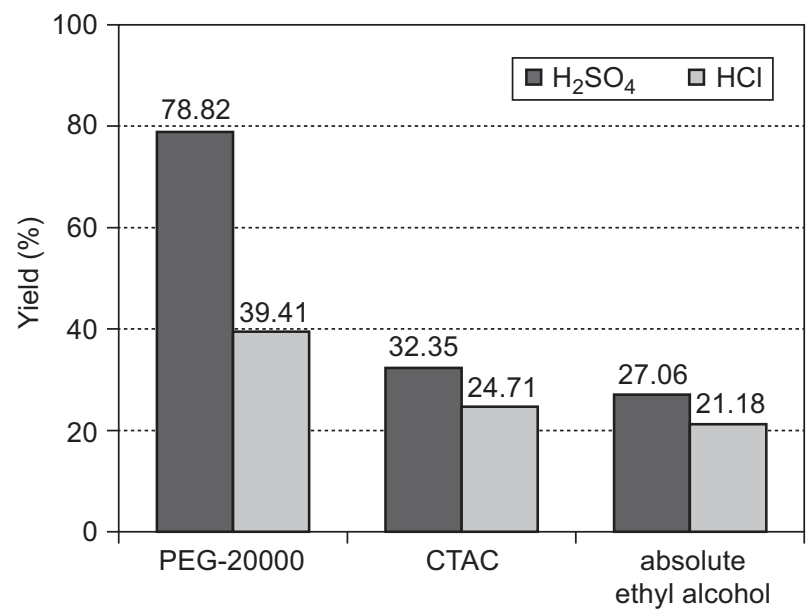

Figure 5. Yield of $\mathrm{MoS}_{2}$ preparation procedures using different acids and surfactants. of the hydroxyl chain. The longer the length of hydroxyl chain, the stronger is the stereo-hindrance. Hence the particles are easier to agglomerate when alcohol is taken as the surfacant. The formation of $\mathrm{MoS}_{2}$ with flower-like structure is probably related to the number of hydroxyl and carbon atoms in the carbon chains.

\section{Yield}

Apart from the morphological characteristics and properties of $\mathrm{MoS}_{2}$, yield is another important parameter that should be evaluated in order to compare, select and improve experimental approaches. Equation 6 can be used to calculate the yield of $\mathrm{MoS}_{2}$ under different conditions.

$$
\text { yield }(\mathrm{Y})=\frac{\text { actual value }(\mathrm{A})}{\text { theoretica } 1 \text { value }(\mathrm{T})} \times 100 \%
$$

The yield of the different $\mathrm{MoS}_{2}$ preparation procedures is compared in Figure 5.

It can be seen that the yield of $\mathrm{MoS}_{2}$ reaches its highest value when polyethylene glycol is used as the surfactant, while the yield using CTAC is intermediate and the yield using absolute ethyl alcohol is the lowest. Further it is evident that generally the yield of $\mathrm{MoS}_{2}$ under sulfuric acid conditions is higher than that of hydrochloric acid conditions. The authors believe that this may be associated with the volatility of hydrochloric acid at high temperature.

\section{CONCLUSIONS}

- Under strongly acidic condition $(\mathrm{pH}=1.0)$, nanoparticles with a spherical structure and diameters of around $100 \mathrm{~nm}$ are obtained by adding polyethylene glycol and hexadecyl trimethyl ammonium chloride. $\mathrm{MoS}_{2}$ nanoparticles with a flower-like structure are obtained when anhydrous ethanol is added. When prepared under sulfuric acid and hydrochloric acid conditions, their diameters are $190 \mathrm{~nm}$ and $70 \mathrm{~nm}$, respectively.

- Using this hydrothermal method, molybdenum disulfide nanoparticles are prepared more easily in sulfuric acid solution than in hydrochloric acid solution. The yield of $\mathrm{MoS}_{2}$ is highest when adding polyethylene glycol.

\section{Acknowledgment}

This work received the support of the Natural Science Foundation of Hebei Province (E2016209331). The authors also gratefully acknowledge the valuable support from Industrial Robot Industry Technology Research Institute of Hebei. 


\section{REFERENCES}

1. Sangita K., Rashi G., Niranjan K., OMP K. (2016): PEGmediated hydrothermal synthesis of hierarchical microspheres of $\mathrm{MoS}_{2}$ nanosheets and their potential for lubrication application. Journal of Industrial and Engineering Chemistry,42, 87-94. doi:10.1016/j.jiec.2016.07.038

2. Zhu W., Wang D.Z., Wu Z.Z., Wang C.L. (2012): Preparation of $\mathrm{MoS}_{2}$ nanocatalysts and their hydrodeoxygenation performances. Petrochemical Technology, 41(6), 658-663. doi:10.3969/j.issn.1000 -8144.2012.06.008

3. Zhai Y.J., Li J.H., Chu X.Y., Xu M.Z., Li X., Fang X., Wei Z.P., Wang X.H. (2015): Preparation and application of molybdenum disulfide nanostructures. Journal of Inorganic Materials, 30(9), 897-905. doi:10.15541/jim20150123

4. Feng Y., Huang S.H., Kang K., Duan X.X. (2011): Preparation and characterization of graphene and few-layer graphene. New Carbon Materials, 26(1), 26-30.

5. Feng Y., Chen K. (2015): Dry transfer of Chemical-VaporDeposition-Grown graphene onto liquid-sensitive surfaces for tunnel junction applications. Nanotechnology, 26, 035301-02. doi:10.1088/0957-4484/26/3/035302

6. Wu C.X., Yang H.B., Li X. Liu S.K. (2007): Anti-friction and anti-wear properties of inorganic fullerene-like molybdenum disulfide. Lubrication and Engineering, 32(7), 118-121. doi:10.3969/j.issn.0254-0150.2007.07.031

7. Zhang L.L., Pu J.B., Zhang G.A., Wang L.P., Xue Q.J. (2015): The preparations of graphene-like molybdenum disulfide and research in tribological properties in high vacuum. Tribology, 35(6), 746-753. doi:10.16078/j.tribology.2015.06.014

8. Wang Q.S., Lan X.Z, Zhou J., Song Y.H., Xing X.D. (2007): Research development on preparation of nanometer molybdenum disulfide by chemical liquid phase reaction. Guangdong Chemical Industry, 34(9), 32-35. doi:10.3969/j. issn.1007-1865.2007.09.010

9. Tenne R. (2002): Inorganic nanotubes and fullerene-like materials. Chemistry, 8(23), 5296-304. doi:10.1002/15213765(20021202)8:23<5296::AID-CHEM5296>3.0.CO;2-P

10. Li W.J., Shi E.W., Ko J.M., Chen Z.Z., Ogino H., Fukuda T. (2003): Hydrothermal synthesis of $\mathrm{MoS}_{2}$ nanowires. Journal of Crystal Growth, 250, 418-442. doi:10.1016/ S0022-0248(02)02412-0

11. Chen J., Li S.L, Xu Q. Koji T. (2002): Synthesis of openended $\mathrm{MoS}_{2}$ nanotubes and the appication as the catalysts of methanation. Chemical Communications, 2(16), 1722-23. doi:10.1039/B205109E
12. Tian Y., He Y., Zhu Y.F. (2004): Low temperature synthesis and characterization of molybdenum disulfide nanotubes and nanorods. Chemistry and Physics, 87(1), 87-90. doi:10.1016/j.matchemphys.2004.05.010

13. Zhang C.F., Wu H.B., Guo Z.P., Lou X.W. (2012): Facile synthesis of carbon-coated $\mathrm{MoS}_{2}$ nanorods with enhanced lithium storage properties. Electrochemistry Communications, 20, 7-10. doi:10.1016/j.elecom.2012.03.039

14. Tian Y.M., Zhao X., Shen L.C., Meng F.Y., Tang L.Q., Deng Y.H., Wang Z.C. (2006): Synthesis of amorphous $\mathrm{MoS}_{2}$ nanospheres by hydrothermal reaction. Materials Letters, 60(4), 527-529. doi:10.1016/j.matlet.2005.09.029

15. Wang L.N., Ma Y., Yang M., Qi Y.X. (2015): Hierarchical hollow $\mathrm{MoS}_{2}$ nanospheres with enhanced electrochemical properties used as an electrode in supercapacitor. Electrochimica Acta, 186, 391-396. doi:10.1016/j.electacta. 2015.10.130

16. Francis L.D., Alvaro M., Miguer J.Y. (2009): Faceted $\mathrm{MoS}_{2}$ nanotubes and nanoflowers. Chemistry and Physics, 118, 392-397. doi:10.1016/j.matchemphys.2009.08.003

17. Zhang H. (2015): Research progress in the preparation and properties of nanometer-sized molybdenum disulfide. China Molybdenum Industry, 39(3), 5-16. doi:10.13384/j. cnki.cmi.1006-2602.2015.03.002

18. Zhao X.L., Li Z., Wang G.X., Xie G.W. (2014): Synthesis characterization of $\mathrm{MoS}_{2}$ microspheres. Journal of Qingdao University of Science and Technology(Natural Science Edition), 35(2), 137-140. doi:10.3969/j.issn.16726987.2014.02.007

19. Feng Y.L., Li P., Zhang J., Xu B., Peng W.T., Song G.C. (2010): Surfactant aided hydrothermal synthesis and characterization of the six-party nanometer Molybdenum disulfide. Journal of Henan University of Science and Technology:Natural Sicence, 31(2), 96-99. doi:10.3969/j. issn.1672-6871.2010.02.026

20.Zou T.Z., Tu J.P., Xia Z.Z., He D.N., Yin G.L. (2005): Precipitation preparation of IF- $\mathrm{MoS}_{2}$ by addition of PEG as surface dispersion agent. Chinese Journal of Inorganic Chemistry, 21(8), 1170-74. doi:10.3321/j.issn:1001-4861. 2005.08.011

21. Zeng Y.F., Jiang X.M., Chen Y.Z. (2001): Effect of surfactants on preparation of $\mathrm{MoS}_{2}$ nanoparticles. Acta Petrolei Sinica(Petroleum Processing Section), 17(4), 55-58. doi:10.3969/j.issn.1001-8719.2001.04.010 